\title{
Designing and Evaluating Interpretable Rule-Based Architecture Under Privacy Constraints: A Framework of Granular Computing
}

\author{
Witold Pedrycz ${ }^{(\bowtie)}$ \\ Department of Electrical and Computer Engineering, University of Alberta, Edmonton, Canada \\ wpedrycz@ualberta.ca
}

\begin{abstract}
In data analytics, system modeling, and decision-making models, the aspects of interpretability and explainability are of paramount relevance as emphasized in numerous studies on explainable Artificial Intelligence (XAI). Those requirements are especially timely when the design of models has to be realized when considering strict requirements of privacy and security.

We advocate that to efficiently address these challenges, it becomes beneficial to engage the fundamental framework of Granular Computing. It is demonstrated that a conceptualization of information granules can be conveniently carried out with the use of information granules (for example, fuzzy sets, sets, rough sets, and alike).

We cover a comprehensive discussion of information granules-oriented design of rule-based architectures. A way of forming condition parts of the rules through unsupervised federated learning is discussed along with algorithmic developments. Strategies of joint and separate learning of condition parts and conclusion parts are outlined. A granular characterization of the model formed by the server vis-a-vis data located at individual clients is presented. It is demonstrated that the quality of the rules at the client's end is described in terms of granular parameters and subsequently the global model becomes represented as a granular model with parameters in the form of information granules of type- 2 .
\end{abstract}

